\title{
NONMETRIC COMPACT SPACES AND NONMEASURABLE PROCESSES
}

\author{
R. M. DUDLEY
}

(Communicated by William D. Sudderth)

\begin{abstract}
Measure theory in compact Hausdorff spaces has some pathological aspects. Some natural functions are nonmeasurable. Others are measurable but have nonmeasurable ranges. Joint measurability is not preserved by the Kakutani-Nelson canonical representation of functions as elements of product spaces, even "almost", for the indicator function of the triangle below the diagonal of the unit square (empirical distribution function for sample size $n=1$ ).
\end{abstract}

Bourbaki [6] defined measures only as Radon measures on locally compact Hausdorff spaces. Some drawbacks of Bourbaki's approach were noted early [21]. The regularity extension, from Baire to regular Borel measures [20, $\S 51-$ 54], is basic for nonmetric compact spaces. The examples given here will show some of the perils of measure theory in such spaces. Specifically, let $I:=[0,1]$, the unit interval. Then $2^{I}$ is the set of all subsets of $I$ (or functions from $I$ into the 2-point space $\{0,1\})$, and $I^{I}$ the set of all functions from $I$ into itself. With the usual product topologies, $2^{I}$ and $I^{I}$ are compact Hausdorff nonmetric spaces.

Let $X$ and $Y$ be two topological spaces, with Borel $\sigma$-algebras generated by their open sets. A function $f$ from $X$ into $Y$ is, as usual, called Borel measurable if $f^{-1}(V)$ is a Borel set in $X$ for every Borel set (equivalently, every open set) $V$ in $Y$. In view of some of the pathology to be noted below, some authors have considered a stronger kind of measurability: given a finite, regular Borel measure $m$ on $X$, a function $f$ from $X$ into $Y$ is said to be Lusin m-measurable if for every $\varepsilon>0$ there is a compact $K \subset X$ such that $m(X \backslash K)<\varepsilon$ and the restriction of $f$ to $K$ is continuous [26, p. 25]. Tjur [28, p. 67] calls a function measurable (given $m$ ) only when it is Lusin $m$-measurable.

There are troubles with some natural, specific functions from $I$ into $2^{I}$ or $I^{I}$. First, consider the function $f(x):=\{x\}$. Each $\{x\}$ belongs to an open set

Received by the editors April 13, 1989.

1980 Mathematics Subject Classification (1985 Revision). Primary 60G05, 60B05, 28A20.

Key words and phrases. Kakutani-Nelson construction, joint measurability.

This research was partly supported by NSF Grant DMS-8803305. 
$U_{x}:=\left\{A \in 2^{I}: x \in A\right\}$ with $\{y\} \notin U_{x}$ for all $y \neq x$. So the relative topology of the range of $f$ is discrete, and $f$ is not measurable from $I$ into $2^{I}$, since not all subsets of $I$ are measurable. Also, the functions $f_{n}$ from $I$ into $I^{I}$ defined by $f_{n}(x)(y):=\max (0,1-n|x-y|)$ are continuous from $I$ into $I^{I}$ and converge pointwise everywhere to the function $f(x):=1_{\{x\}}$ which, as just noted, is not measurable $[12 ; 17$, p. 96].

The functions $f_{n}$ are Lusin measurable from $I$ into $I^{I}$ for an arbitrary measure $m$, and where the compact set $K$ in each case can be taken as the whole domain space $I$. So the use of Lusin measurability (as defined and referenced above) does not prevent the nonmeasurability of sequential limits.

The second example, which the rest of the note will treat, is the function $F_{1}(x):=[x, 1]$. Now $F_{1}$ is Borel measurable from $I$ into $2^{I}$, since any union of nondegenerate intervals (closed or open at either end, but not reducing to singletons) can be written as a countable disjoint union, which is Borel in $I$. Let $M_{-}$be the range of $F_{1}$ in $2^{I}$. Let $\left.\left.G(x):=\right] x, 1\right]$ for $0 \leq x \leq 1$ and let $M_{+}$be the range of $G$ in $2^{I}$. Let $M=M_{-} \cup M_{+}$. Then $M$ is compact in $2^{I}$.

A Borel measurable function from a Borel set in a complete separable metric space into a separable metric space $Y$ has a range which is not necessarily Borel, but is analytic, so that it is universally measurable, i.e. measurable for the completion of any Borel measure on $Y$ (e.g. [7, §8.4] or [17, §13.2]). In general, however, given a measure space $(X, m)$ and a measurable function $g$ from $X$ into a measurable space $(Y, \widetilde{A})$, the range of $g$ may not be measurable for the image measure $m \circ g^{-1}$ on $\widetilde{A}$ ("image measure catastrophe": [26, p. 30]); it will be if $g$ is Lusin measurable. If a measure is purely atomic, all sets are measurable for it. A measure will be called nonatomic if it has no atoms. Here is a case where the range of a Borel function from $[0,1]$ into a compact Hausdorff space is "universally nonmeasurable".

Proposition 1. Let $\gamma$ be any nonatomic regular Borel measure on $2^{I}$. Then $M_{-}$ and $M_{+}$both have inner measure 0 for $\gamma$. So if $\gamma(M)>0$ then $M_{-}$and $M_{+}$ are both nonmeasurable for $\gamma$.

Proof. Let $C$ be a compact subset of $M_{-}$, so $C=\{[x, 1]: x \in D\}$ for some set $D \subset I$. Then $D$ cannot contain any strictly decreasing sequence $x_{n} \downarrow x$, whose limit would yield $] x, 1] \in M_{+}$. So for each $x$ in $D$, except for $x_{0}:=\sup D$ if $x_{0} \in D$, there is a next larger member $g(x) \in D$. Let $g\left(x_{0}\right)=1$. Then $D$ is countable, since for each positive integer $n$ the subset of $D$ where $g(x)-x>$ $1 / n$ is finite. (Also, $M_{-}$is homeomorphic to $[0,1]$ with the topology induced by the Sorgenfrey [27] topology on $\mathbf{R}$ having a base of all half-open intervals ]$a, b]$. There it is known that a set $D$ without accumulation points from the right is countable [23, p. 59].) Since singletons have $\gamma$ measure 0 , we have $\gamma(C)=0$. So by regularity, $M_{-}$has inner measure 0 for $\gamma$, and likewise for $M_{+}$. Q.E.D. 
Since the last proof shows that $M_{-}$has no uncountable compact subsets, we get, as noted in [28, p. 198] for $m=$ Lebesgue measure $\lambda$,

Proposition 2. $F_{1}$ is not Lusin m-measurable from $I$ into $2^{I}$ for any nonatomic, nonzero Borel measure $m$ on $I$.

For any $A \in 2^{I}$ let $\tau(A):=\inf \{q: q$ rational, $q \in A\}$. Then $\tau$ is Baire measurable from $2^{I}$ onto $I .(\rho(A):=\inf \{t: t \in A\}$ is not Baire; $\rho$ and $\tau$ are upper semicontinuous, as a referee pointed out.) For $0 \leq x \leq 1, \quad \tau([x, 1])=$ $\tau(] x, 1])=x$. For the case where $\gamma$ is the image of $\lambda$ on $I$ by $F_{1}$, the following fact is mentioned, with a sketch of a quite different proof, in [28, $\mathrm{p}$. 198].

Proposition 3. For any nonatomic regular Borel measure $\gamma$ on $2^{I}$ with $\gamma(M)>$ 0 , the function $f \mapsto f(\tau(f))$ from $2^{I}$ onto $\{0,1\}$ is not measurable for $\gamma$.

Proof. We have $f(\tau(f))=1$ if $f \in M_{-}$and $f(\tau(f))=0$ if $f \in M_{+}$. So the result follows from Proposition 1.

Let $T$ be any set and $(\Omega, \widetilde{S}, P)$ a probability space. For each $t \in T$ let $X_{t}$ be a measurable real function on $\Omega$. In other words, $X_{t}, t \in T$, is a stochastic process. Let $\widetilde{B}$ be a $\sigma$-algebra of subsets of $T$. Then the process is called measurable if $(t, \omega) \mapsto X_{t}(\omega)$ is measurable for the product $\sigma$-algebra. I will call $X_{t}$ almost measurable if there is a subset $W \subset \Omega$ with $P(W)=1$ such that the process is measurable on $T \times W$. If $T$ is a subset of the line with positive Lebesgue measure, I will call the process Doob measurable if $(t, \omega) \mapsto X_{t}(\omega)$ is measurable for the completion of the product measure $\lambda \times P[8 ; 9, \mathrm{p} .60]$.

If $\tau$ is a measurable function from $\Omega$ into $T$ and $X_{t}$ is an almost measurable process, then $X_{\tau}: \omega \mapsto X_{\tau(\omega)}(\omega)$ is measurable (for the completion of $P$ on $\Omega$ ). A stochastic process $X_{t}, t \in T$, will be called canonical if $\Omega$ is itself a set of functions $f$ on $T$ and $X_{t}(f) \equiv f(t)$. Proposition 3 then gives

Proposition 4. For any $\gamma$ on $2^{I}$ as in Proposition 3, the canonical process defined by $\gamma$ is not almost measurable.

Kakutani [22] defined product probabilities on infinite products of compact Hausdorff spaces. For any real-valued stochastic process $X_{t}, t \in T$, Nelson [24] defined a corresponding canonical process as follows. Embed $\mathbf{R}$ in the compact space $\overline{\mathbf{R}}:=[-\infty, \infty]$ of extended real numbers. Then there is a regular Borel probability measure $P$ on the space $\overline{\mathbf{R}}^{T}$ of all functions from $T$ into $\overline{\mathbf{R}}$ such that for any finite $n$, any $t_{i}:=t(i) \in T, i=1, \ldots, n$, and any Borel sets $B_{i} \subset \mathbf{R}$,

$$
\operatorname{Pr}\left\{X_{t(i)} \in B_{i}, i=1, \ldots, n\right\}=P\left\{f: f\left(t_{i}\right) \in B_{i}, i=1, \ldots, n\right\} .
$$

Nelson showed that certain spaces of functions such as $C[0,1]$ and the space of real functions on $I$ with right and left limits everywhere are Borel sets in $\overline{\mathbf{R}}^{T}$. Tjur [28] proposed to base probability on Randon measures, treating stochastic processes by the Kakutani-Nelson construction [28, p. 202]. 
Doob [8] asked whether (Doob) measurability of a process $X_{t}$ would carry over to its corresponding canonical process. In $[12,13,14 ; 17$, Appendix E] there is a counterexample, assuming the continuum hypothesis, where $T=H$, a separable, infinite-dimensional Hilbert space, for the isonormal Gaussian process on $H$ with mean 0 and covariance given by the inner product. A particular (Gaussian) measure on $H$ took the place of Lebesgue measure on $I$; any such measure is an image of Lebesgue measure by a Borel measurable transformation, e.g. [17, Theorem 11.7.5], which can be taken to be $1-1$ [25, $\S 2 \# 7]$. The proof of nonmeasurability was rather difficult and unintuitive.

Variables $X_{\tau}$ for random times $\tau$ such as stopping times are crucial in the theory of some classes of processes, including Markov processes (e.g. [19, p. 98]). The measurability of the process evaluated at a random time (or a random point in a more general parameter space $T$ ) is one of the main benefits of measurability.

Doob [8] asked, in effect, whether $(t, f) \mapsto f(t)$ is Doob measurable for Nelson's canonical process if $X_{t}$ is Doob measurable. But for a Doob measurable process, $X_{\tau}$ need not be measurable since the graph $\{(t, \omega): t=\tau(\omega)\}$ has $\lambda \times P$ measure 0 , so the process can be arbitrary on the graph. We can then ask whether the canonical process is almost measurable if $X_{t}$ is. For a process with continuous paths, taking $W=C[0,1]$ in $\overline{\mathbf{R}}^{I}$, the corresponding canonical process is almost measurable since $W$ is a Borel set [24]. But Proposition 4 implies the following:

Proposition 5. For the process $X_{t}(\omega):=1_{\{\omega \leq t\}}$ on $I \times I$, and Lebesgue measure on both copies of $I$, the canonical process on $I^{I}$ is not almost measurable.

The function $F_{1}$ from $I$ into $2^{I}$ is not only elementary but important as an example of an empirical distribution function. Such functions have been considered also in the supremum norm, where the range of $F_{1}$ is nonseparable and $F_{1}$ is again not Borel measurable, and not Lusin measurable (cf. Proposition 2 ). Yet one wants to work with $F_{1}$ and "empirical processes" more generally that share some of the same measurability problems.

Recall that $D[0,1]$ is the set of real functions on $I$ continuous from the right (and from the left at 1) and with limits from the left. Since $D[0,1] \cap M=M_{-}$ it follows from Prop. 1 that $D[0,1]$ is nonmeasurable in $\overline{\mathbf{R}}^{I}$ for certain Borel measures and so at any rate is not Borel. $F_{1}$ has values in $D[0,1]$, which can be given a complete, separable metric (Skorohod topology) for which convergence to a continuous function is equivalent to convergence in the supremum norm (e.g. [5, Chapter 3]). But $D[0,1]$ seems not to extend to enough generality. The theory of empirical processes has been treating nonmeasurable real-valued functions and their upper and lower integrals [10, 11, 15, 18]. More recently, using an idea of J. Hoffmann-Jørgensen, the upper and lower integrals are taken over a probability space $\Omega$, without trying to define a distribution for processes such as $F_{1}$ on a function space [16]. In other words, the idea of a "canonical" 
representation of a stochastic process, which runs into difficulties with measurability in compact Hausdorff spaces, also has problems in metric spaces. Instead, the theory encompassing possibly nonmeasurable functions seems to work well enough $[1,2,3,4,16]$.

\section{REFERENCES}

1. N. T, Andersen, The central limit theorem for non-separable valued functions, Z. Wahrsch. verw. Geb. 70 (1985), 445-455.

2. $\ldots$ The calculus of nonmeasurable functions and sets, Various Publ. Series No. 36 (1985), Math. Inst., Aarhus Univ.

3. N. T. Andersen and V. Dobrić, The central limit theorem for stochastic processes, Ann. Prob. 15 (1987), 164-177.

4. 287-303.

5. P. Billingsley, Convergence of probability measures, Wiley, New York, 1968.

6. N. Bourbaki, Intégration, Hermann, Paris, Chaps. 1-4, 1952, 2nd ed., 1965; Chap. 5, 1956, 2nd ed., 1965; Chap. 6, 1959; Chaps. 7-8, 1963; Chap. 9, 1969.

7. D. L. Cohn, Measure theory, Birkhäuser, Boston, 1980.

8. J. L. Doob, Probability in function space, Bull. Amer. Math. Soc 53 (1947), 15-30.

9. __ Stochastic processes, Wiley, New York, 1953.

10. R. M. Dudley, Weak convergence of probabilities on nonseparable metric spaces and empirical measures on Euclidean spaces, Illinois J. Math. 10 (1966), 109-126.

11. __ Measures on non-separable metric spaces, Illinois J. Math 11 (1967), 449-453.

12. __ On measurability over product spaces, Bull. Amer. Math. Soc. 77 (1971), 271-274.

13. $\_$A counterexample on measurable processes, Proc. 6th Berkeley Symp. Math. Statist. Prob. 2 (1972), 57-66.

14. Correction, Ann. Probab. 1 (1973), 191-192.

15. __ A course on empirical processes, Ecole d'été probabilités de St.-Flour, 1982; Lecture Notes in Math. 1097, (1984) Springer-Verlag, 1-142.

16. _ An extended Wichura theorem, definitions of Donsker class, and weighted empirical distributions, Probability in Banach Spaces V (Proc. Conf. Medford, 1984), Lecture Notes in Math. 1153, (1985) Springer, 141-178.

17. __ Real analysis and probability, Brooks/Cole, Pacific Grove, CA, 1989.

18. R. M. Dudley and W. Philipp, Invariance principles for sums of Banach space valued random elements and empirical processes, Z. Wahrsch. verw. Geb. 62 (1983), 509-552.

19. E. B. Dynkin, Markov processes I, transl. by J. Fabius et al., Academic Press, New York; Springer, Berlin, 1965.

20. P. R. Halmos, Measure theory, Van Nostrand, Princeton, 1950.

21. _ـ Review of [6, Chaps. 1-4], Bull. Amer. Math. Soc. 59 (1953), 249-255.

22. S. Kakutani, Notes on infinite product spaces II, Proc. Imp. Acad. Tokyo 19 (1943), 184-188.

23. J. L. Kelley, General topology, Van Nostrand, Princeton, 1955.

24. E. Nelson, Regular probability measures on function space, Ann. Math. 69 (1959), 630-643.

25. V. A. Rokhlin, On the fundamental ideas of measure theory, Mat. Sb. (N.S.) 25 (1949), 107-150 (in Russian); Amer. Math. Soc. Transl. 71 (1952), 1-54.

26. L. Schwartz, Radon measures on arbitrary topological spaces, Oxford Univ. Press, 1973.

27. R. H. Sorgenfrey, On the topological product of paracompact spaces, Bull. Amer. Math. Soc. 53, 631-632.

28. T. Tjur, Probability based on Radon measures, Wiley, Chichester, 1980.

Department of Mathematics, Massachusetts institute of Technology, Cambridge, MASSACHUSETTS 02139 